\title{
Distinct Gene Expression Profile Distinguishes Increased Metabolic Activity in Spontaneously Hyperactive Rats While Sedentary from That Induced by Exercise
}

\author{
Manami Abe1, Yuki Matsuo', Akiko Harada-Sukeno1, Takayuki Uchida1, Kanako Kitahata1, \\ Chisato Tomida ${ }^{1}$, Katsuya Hirasaka ${ }^{2}$, Shigetada Teshima-Kondo ${ }^{1,3}$, Nagakatsu Harada ${ }^{4}$, \\ Yutaka Nakaya', Hiroshi Sakaue ${ }^{4}$, Reiko Nakao1, Takeshi Nikawa1 $^{*}$ \\ ${ }^{1}$ Departments of Nutritional Physiology, Institute of Biomedical Sciences, Tokushima University Graduate School, Tokushima, \\ Japan \\ ${ }^{2}$ Laboratory of Fishery Nutritional Science, Graduate School of Fisheries and Environmental Sciences, Nagasaki University, \\ Nagasaki, Japan \\ ${ }^{3}$ Department of Medical Nutrition, Graduate School of Comprehensive Rehabilitation, Osaka Prefecture University, Osaka, Japan \\ ${ }^{4}$ Departments of Nutrition and Metabolism, Institute of Biomedical Sciences, Tokushima University Graduate School, Tokushima, \\ Japan \\ Email: *nikawa@tokushima-u.ac.jp
}

How to cite this paper: Abe, M., Matsuo, Y., Harada-Sukeno, A., Uchida, T., Kitahata, K., Tomida, C., Hirasaka, K., TeshimaKondo, S., Harada, N., Nakaya, Y., Sakaue, H., Nakao, R. and Nikawa, T. (2018) Distinct Gene Expression Profile Distinguishes Increased Metabolic Activity in Spontaneously Hyperactive Rats While Sedentary from That Induced by Exercise. Advances in Biological Chemistry, 8, 1-14.

https://doi.org/10.4236/abc.2018.81001

Received: December 19, 2017

Accepted: February 2, 2018

Published: February 5, 2018

Copyright $\odot 2018$ by authors and Scientific Research Publishing Inc. This work is licensed under the Creative Commons Attribution International License (CC BY 4.0). http://creativecommons.org/licenses/by/4.0/

\begin{abstract}
The Spontaneously-Running Tokushima Shikoku (SPORTS) strain is an original line derived from Wistar rats, which spontaneously runs $>6 \mathrm{~km} /$ day on wheels, and has better glucose tolerance and less fat than Wistar rats. However, the molecular mechanism that contributes to the increased metabolic activity in SPORTS rats is unknown. The present study aimed to characterize the gene expression profiles of skeletal muscles in SPORTS rats housed under sedentary (SED) conditions. We found that the expression levels of genes encoding mitochondrial respiratory chain enzymes such as ATP synthase 6 (mt-Atp6) and cytochrome c oxidase subunit $6 c$ (Cox6c), were higher in the soleus (SOL) muscles of SED SPORTS than in SED Wistar rats. The ratio of type IIa myofibers was higher and glucose tolerance was better in SED SPORTS than in Wistar rats that were sedentary and trained daily on treadmills, respectively. We then investigated candidate genes that might contribute to the better glucose tolerance of SED SPORTS rats using DNA microarray analysis. Among 116 upregulated genes in the SOL muscles of SED SPORTS rats, only 19 were also increased in trained Wistar rats. We focused on v-erb-b2 erythroblastic leukemia viral oncogene homolog 3 (Erbb3), which
\end{abstract}


was associated with glucose transport in myocytes, and found higher expression levels in the SOL muscles of SED SPORTS than in SED Wistar rats. The SOL muscles of SED SPORTS rats also contained more activity of $\beta$-hydroxyacylCoA dehydrogenase, a key enzyme of $\beta$-oxidation, indicating enhanced lipid oxidation. These findings suggest that increased metabolic activity in skeletal muscle (especially the SOL muscle) of SPORTS rats is congenital and that gene expression profiles of SPORTS rats and Trained Wistar rats are different.

\section{Keywords}

Mitochondria, Myofiber Type, Microarray, Metabolism

\section{Introduction}

Morishima-Yamato et al. established an original line of hyperactive rats derived from the Wistar strain named Spontaneously-Running-Tokushima-Shikoku (SPORTS) rats [1]. This line has the unique characteristic of indulging in extreme amounts of voluntary exercise; when housed with a running-wheel, these rats will voluntarily run about six-fold further than control Wistar rats. Furthermore, SPORTS rats housed without running-wheels have unique metabolic characteristics. Hattori et al. reported significantly lower blood glucose levels in sedentary SPORTS rats than in control Wistar rats, as well as significantly less abdominal fat and plasma triglyceride than control Wistar rats when fed with a high fat and sucrose diet [2]. These findings suggest enhanced lipid utilization or hydrolysis in SPORTS rats, even when sedentary.

Skeletal muscle comprises the largest organ in body and contributes to 30\% $40 \%$ of the resting metabolic rate in adult rats. It is a major site of glucose and fatty acid oxidation, which accounts for $\sim 80 \%$ of insulin-stimulated glucose uptake [3]. The most fundamental component of muscle, myosin, determines the rate of contraction and the resulting metabolic demands of each type of muscle fiber. The four main muscle myosin heavy chains in order of increasing rates of ATPase activity, are type I slow/oxidative, type IIa fast/oxidative, type IIx fast/ intermediate, and type IIb fast/glycolytic, but also increasing fatigability [4]. We postulated that metabolic activity was increased in the skeletal muscles of sedentary SPORTS rats and accompanied by a shift in myofiber type to allow effective glucose and lipid usage and adaptation to extreme running activity. Here, we comprehensively analyzed gene expression profiles in the skeletal muscle of SPORTS rats, with focus on genes associated with energy metabolism.

\section{Materials and Methods}

\subsection{Animals}

We bred and maintained SPORTS rats as described [1]. Seven-week-old male Wistar (Japan SLC Inc., Hamamatsu, Japan) and SPORTS rats were habituated 
and fed with a normal CE-2 diet (Clea Japan Inc., Tokyo, Japan) ad libitum in a room at $23^{\circ} \mathrm{C} \pm 1{ }^{\circ} \mathrm{C}$ under a 12 -h light-12-h dark cycle. All rats were individually housed in cages without running wheels to mimic sedentary (SED) conditions. Wistar rats were randomly assigned to groups that either remained sedentary (SED Wistar) or were trained by moderate daily running on treadmills for four weeks (Trained Wistar). The soleus (SOL) and extensor digitorum longus (EDL) muscles were isolated, frozen, and stored at $-80^{\circ} \mathrm{C}$. These experiments proceeded according to the international guiding principles for biomedical research involving animals and were approved by the Committee for Experimental Animals at the University of Tokushima.

\subsection{Training Procedure}

Groups of rats were exercised by running for a total of $120 \mathrm{~min} /$ day on custombuilt rodent treadmills with one animal per lane. The rats initially ran at $6 \mathrm{~m} /$ $\min$ for $5 \mathrm{~min}$, followed by $10 \mathrm{~m} / \mathrm{min}$ for $10 \mathrm{~min}, 13 \mathrm{~m} / \mathrm{min}$ for $15 \mathrm{~min}$, and 16 $\mathrm{m} / \mathrm{min}$ for $90 \mathrm{~min}$.

\subsection{Electrophoretic Separation of Myosin Heavy Chain (MyHC) Isoforms}

Soleus and EDL muscles dissected from SED Wistar and SED SPORTS rats were homogenized in $20 \mathrm{mM}$ Tris- $\mathrm{HCl}$ buffer ( $\mathrm{pH}$ 6.8) containing $250 \mathrm{mM}$ sucrose, $100 \mathrm{mM}$ potassium chloride $(\mathrm{KCl}), 5 \mathrm{mM}$ EDTA, $100 \mu \mathrm{M}$ phenylmethylsulfonyl fluoride (PMSF), $10 \mathrm{ng} / \mathrm{mL}$ leupeptin and $10 \mathrm{ng} / \mathrm{mL}$ pepstatin A. After centrifugation, sedimented pellets were resuspended in $20 \mathrm{mM}$ Tris- $\mathrm{HCl}$ buffer ( $\mathrm{pH}$ 6.8) containing $175 \mathrm{mM} \mathrm{KCl,} 2 \mathrm{mM}$ EDTA, 0.5\% Triton X-100, $50 \mu \mathrm{M}$ PMSF, $5 \mathrm{ng} /$ $\mathrm{mL}$ leupeptin and $5 \mathrm{ng} / \mathrm{mL}$ pepstatin A. Following centrifugation, sedimented pellets were resuspended in $20 \mathrm{mM}$ Tris- $\mathrm{HCl}$ buffer $(\mathrm{pH} 7.0)$, containing 150 $\mathrm{mM} \mathrm{KCl}, 100 \mu \mathrm{M}$ PMSF, $10 \mathrm{ng} / \mathrm{mL}$ leupeptin and $10 \mathrm{ng} / \mathrm{mL}$ pepstatin A. Total protein concentrations were assayed using Bio-Rad Protein Assays (Bio-Rad Laboratories, Richmond, CA, USA). Proteins (100 ng) were separated by highresolution sodium dodecyl sulfate (SDS)-polyacrylamide gel electrophoresis to assess the content of MyHC isoform as described by Mizunoya et al. [5] and visualized by silver staining using a kit (Wako Pure Chemical Industries, Ltd., Osaka, Japan). Bands were quantified by densitometry using Image J software (National Institutes of Health, Bethesda, MD, USA) and MyHC isoforms were identified according to their apparent molecular masses as indicated by migration rates $(\mathrm{I}>\mathrm{IIb}>\mathrm{IIx}>\mathrm{IIa})$.

\subsection{Glucose Tolerance Test}

Glucose tolerance tests proceeded as described [2]. Rats were fasted for $16 \mathrm{~h}$ and then injected intraperitoneally with glucose $(1 \mathrm{~g} / \mathrm{kg}$ body weight). Blood was collected from the tail vein at $0 \mathrm{~min}$, and at 15,30, 60 and $120 \mathrm{~min}$ after injection to measure blood glucose and insulin levels using Glutestsensor (Sanwa Kagaku 
Kenkyusho Co. Ltd., Nagoya, Japan) and enzyme-linked immunosorbent assay kits (Morinaga Institute of Biological Science, Yokohama, Japan), respectively.

\subsection{DNA Microarray Analysis}

Total RNA was isolated from the SOL muscles of SED or Trained Wistar rats and SED SPORTS rats. Total RNA $(1 \mu \mathrm{g})$ was analyzed using DNA microarrays (Affimetrix, Santa Clara, CA, USA), as described [6]. Gene expression was analyzed using Subio Platform Software (Subio, Tokyo, Japan) [7].

\subsection{Real-Time Reverse Transcription-Polymerase Chain Reaction (RT-PCR)}

Total mRNA was extracted from skeletal muscles using ISOGEN (Nippon Gene, Osaka, Japan) detergent then cDNA was synthesized and quantitative PCR proceeded using a Real time PCR system (Applied Biosystems, Foster City, CA, USA) and $10 \mu \mathrm{L}$ of SYBR-Green Master Mix (Applied Biosystems Ltd., Woolston, Cheshire, UK). The ratio of target to glyceraldehyde-3-phosphate dehydrogenase (Gapdh) expression was calculated. Table 1 lists the primer sequences used for amplification.

Table 1. Primer sets used in this study.

\begin{tabular}{|c|c|c|c|}
\hline Target & & Sequence & Length (bp) \\
\hline \multirow{3}{*}{ D-loop } & S & 5'-CCTCCGTGAAATCAACAACC-3' & \multirow{3}{*}{148} \\
\hline & & & \\
\hline & AS & 5’-TAAGGGGAACGTATGGACGA-3' & \\
\hline \multirow{3}{*}{ Rn18s } & S & 5'-CATTCGAACGTCTGCCCTA-3' & \multirow{3}{*}{137} \\
\hline & & & \\
\hline & AS & 5'-CCTGCTGCCTTCCTTGGA-3' & \\
\hline \multirow{3}{*}{$M t-A t p 6$} & S & 5'-CCGACTACACTCATTTCAACACT-3' & \multirow{3}{*}{91} \\
\hline & & & \\
\hline & AS & 5'-TAGGGCTCAGGTTCGTCCTT-3' & \\
\hline \multirow{3}{*}{ Cox6c } & $S$ & 5'-TGCCGAAACCACAGATGC-3' & \multirow{3}{*}{112} \\
\hline & & & \\
\hline & AS & 5'-TCAGCCACGCCAAACTTAT-3' & \\
\hline \multirow[b]{2}{*}{$P g c-1 \alpha$} & $S$ & 5'-GTGTGGAACTCTCTGGAACTGC-3' & \multirow[b]{2}{*}{139} \\
\hline & AS & 5'-GCCCTCTTTGTTGGTGGTG-3' & \\
\hline \multirow{3}{*}{$P g_{\mathcal{C}-1 \beta}$} & & & \multirow{3}{*}{145} \\
\hline & S & 5'-TGGATGAGCTTTCACTGCTG-3' & \\
\hline & AS & 5'-GTGCCATCCACCTTGACAC-3' & \\
\hline \multirow{3}{*}{ Erbb3 } & S & 5'-CGTCCAGCATCAGAGTCATC-3' & \multirow{3}{*}{135} \\
\hline & & & \\
\hline & AS & 5'-CTGCGAATGGTAGGCACT-3' & \\
\hline \multirow[b]{2}{*}{ Gapdh } & S & 5'-CGTGTTCCTACCCCCAATGT-3' & \multirow[b]{2}{*}{74} \\
\hline & AS & 5'-ATGTCATCATACTTGGCAGGTTTCT-3' & \\
\hline
\end{tabular}

S, sense primer; AS, antisense primer.

D-loop is the initial region of mitochondrial DNA replication, so its copy 
number can indicate the level of mitochondrial DNA (mtDNA) replication [8]. Total DNA was extracted from SOL muscles using QIAamp DNA Mini kits (Qiagen, Tokyo, Japan) to determine mitochondrial contents and then fragments of mtDNA D-loop region were amplified by quantitative PCR. Amounts are shown relative to those of nuclear $18 \mathrm{~S}$ ribosomal RNA $(R n 18 s)$.

\subsection{Biochemical Analyses}

The amount of glycogen in skeletal muscle was determined using the anthronesulfuric acid method as described [9]. Total muscle $\beta$-hydroxyacyl-CoA dehydrogenase ( $\beta$-HAD) activity was measured as described [10]. Skeletal muscle ( $50 \mathrm{mg}$ ) was homogenized in $100 \mu \mathrm{L}$ of $20 \mathrm{mM}$ phosphate buffer ( $\mathrm{pH} 7.4$ ). Samples were then incubated in $100 \mathrm{mM}$ triethanolamine- $\mathrm{HCl}$ buffer $(\mathrm{pH} 7.0)$, and the reaction was started by adding $0.1 \mathrm{mM}$ acetoacetyl-CoA. Absorbance was measured at $340 \mathrm{~nm}$ and protein concentrations were determined using BCA protein assay kits (Thermo Fisher Scientific, Rockford, IL, USA).

\subsection{Statistical Analysis}

All data were analyzed using SPSS software (release 6.1; SPSS Japan, Tokyo) and are expressed as means \pm standard deviation (SD). Values derived from SED SPORTS and SED Wistar rats were assessed using Student's $t$-test. Blood glucose and serum insulin levels were evaluated using two-way ANOVA followed by Tukey-Kramer multiple comparison tests. Differences were considered significant at $P<0.05$.

\section{Results}

\subsection{Gene Expression in the Respiratory Chain in Sports Rat Muscle}

We postulated that the hyperactivity in SPORTS rats resulted from upregulated enzymes of the mitochondrial respiratory chain [11]. We measured levels of D-loop gene expression in the SOL and EDL muscles of SED SPORTS and SED Wistar rats to estimate mitochondrial biogenesis since D-loop copy number is indicative of mtDNA replication intensity [8]. Less D-loop was expressed in the SOL muscles of SED SPORTS than SED Wistar rats, but essentially identical amounts were found in EDL muscles from SPORTS and Wistar rats (Figure 1 (a)). The mRNA expression levels of genes encoding key enzymes of the mitochondrial respiratory chain, namely ATP synthase 6 (Mt-Atp6) and cytochrome c oxidase subunit $6 c(C O X 6 c)$ [12], were significantly higher in SOL muscles from SPORTS than Wistar rats (Figure 1(b) and Figure 1(c)). More Mt-Atp6 and Cox $6 \mathrm{cmRNA}$ was also expressed in the EDL muscles of SPORTS than Wistar rats, but the difference did not reach significance (Figure 1(b) and Figure $1(\mathrm{c})$ ). Peroxisome proliferator-activated receptor $\gamma$ coactivator 1 (PGC-1) transcriptionally modulates the expression of genes encoding respiratory chain 

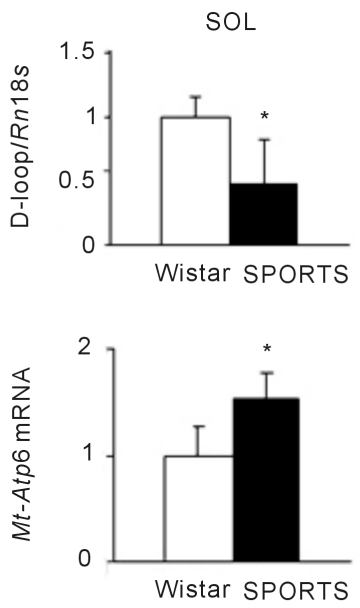

(c)

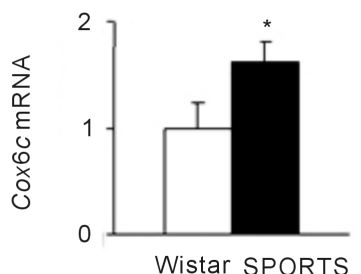

(d)

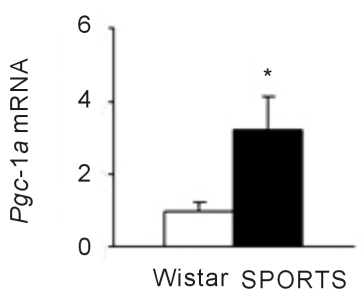

(e)



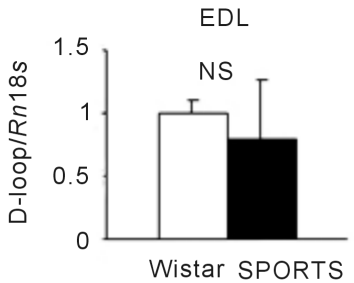
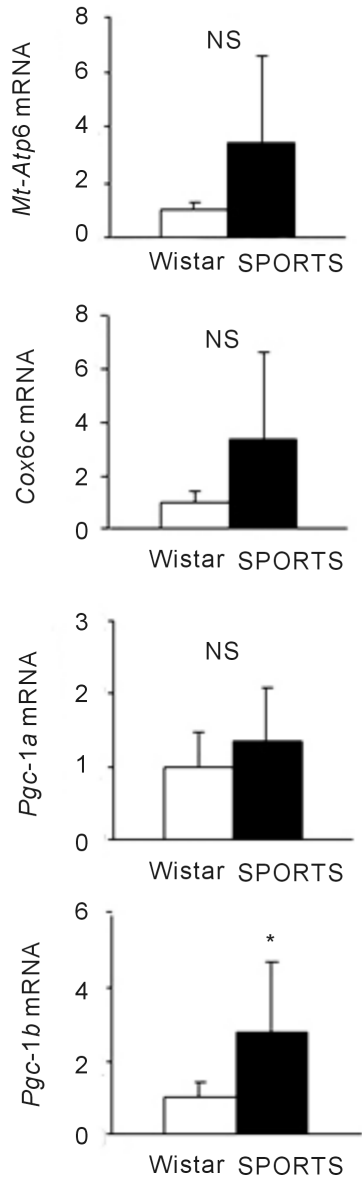

Figure 1. Expression profiles of genes encoding mitochondrial respiratory chain enzymes. (a) Mitochondrial DNA expression in SOL and EDL muscles of sedentary SPORTS and Wistar rats are shown as a ratio between D-loop and 18S rRNA. (b)-(e) Messenger RNA expression of genes encoding mitochondrial enzymes related to respiratory chain. Data are means \pm SD ( $n=7$ per group). Expression for sedentary Wistar rats is expressed as 1.0. ${ }^{*} P<0.05$ compared with Wistar rats. EDL: extensor digitorum longus muscle; NS: not significant; SOL: soleus muscle.

enzymes, including Mt-Atp6 and COX6c [13]. The amount of Pgc-1a (also known as Ppargcla) mRNA was 3-fold higher in the SOL muscles of SPORTS, than Wistar rats, but did not differ between EDL muscles from these rats (Figure 1(d)). On the other hand, the expression of $P g c-1 \beta$ (also known as Ppargc1b) mRNA was essentially identical in the SOL muscles of SPORTS and Wistar rats, but significantly higher in the EDL muscles of SPORTS than Wistar rats (Figure $1(\mathrm{e})$ ). 


\subsection{Expression of Myosin Heavy Chain Isoforms in SPORTS Rat Muscle}

The upregulated expression of $P g c-1 \alpha$ and $P g c-1 \beta$ in SPORTS rat SOL and EDL muscles, respectively, led to an investigation of myofiber types in SPORTS rats. The ratio of myosin heavy chain isoforms was electrophoretically determined in the SOL (Figure 2(a)) and EDL (Figure 2(b)) muscles of SED SPORTS and SED Wistar rats. The composition of SOL muscle fibers (mainly types I and IIa) did not differ between SED SPORTS and SED Wistar rats (Figure 2(c)). In contrast, the ratio of type IIa fibers was significantly higher in EDL muscles (usually mainly fast-twitch fibers) from SED-SPORTS, than from SED Wistar rats (Figure 2(d)), whereas the ratio of type IIx fibers did not significantly change in EDL muscles. The ratios of types I and IIb fibers were slightly decreased in the EDL muscles of SPORTS, compared with Wistar rats (Figure 2(d)).

\subsection{Glucose Tolerance in SPORTS Rats}

We evaluated glucose tolerance in SED Wistar, Trained Wistar and SED SPORTS rats. Consistent with previous findings [2], blood glucose levels were significantly lower in SED SPORTS, than in SED Wistar rats at 15, 30, and 60 min after an intraperitoneal injection of glucose (Figure 3(a)). Although moderate treadmill running for four weeks improved glucose tolerance in Wistar rats, blood glucose concentrations were higher in Trained Wistar than in SED

(a)

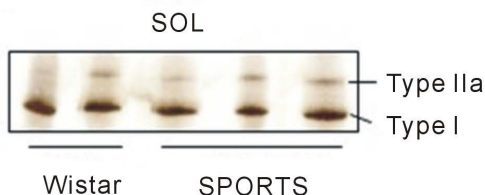

(c)

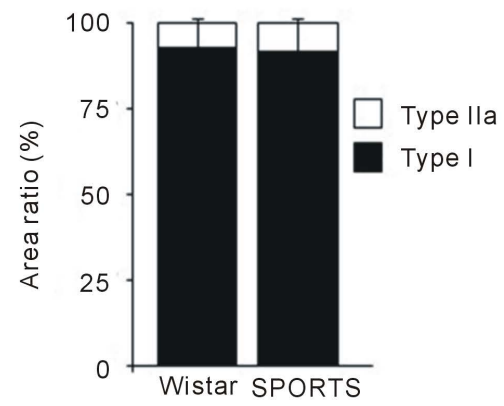

(b)

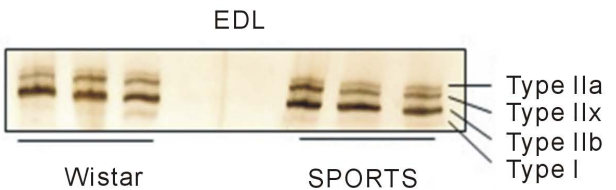

(d)

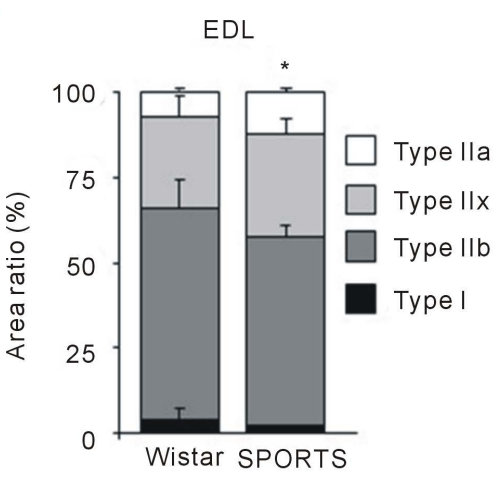

Figure 2. Characterization of myofiber types. (a), (b) Extracted muscle protein from sedentary SPORTS and Wistar rats separated by SDS-polyacrylamide gel electrophoresis and visualized by silver staining. (c), (d) Bands were quantified by densitometry and averaged ratios of myosin types to total area are shown as graphs. Data are means \pm SD $(n=4) .{ }^{*} P<$ 0.05 compared with Wistar rats. EDL: extensor digitorum longus muscle; SOL: soleus muscle. 


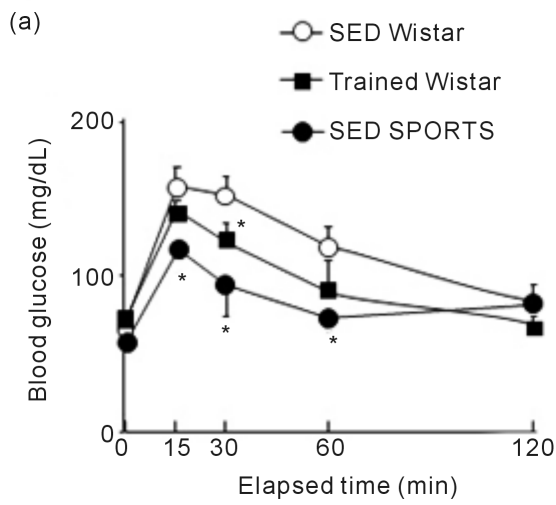

(b)
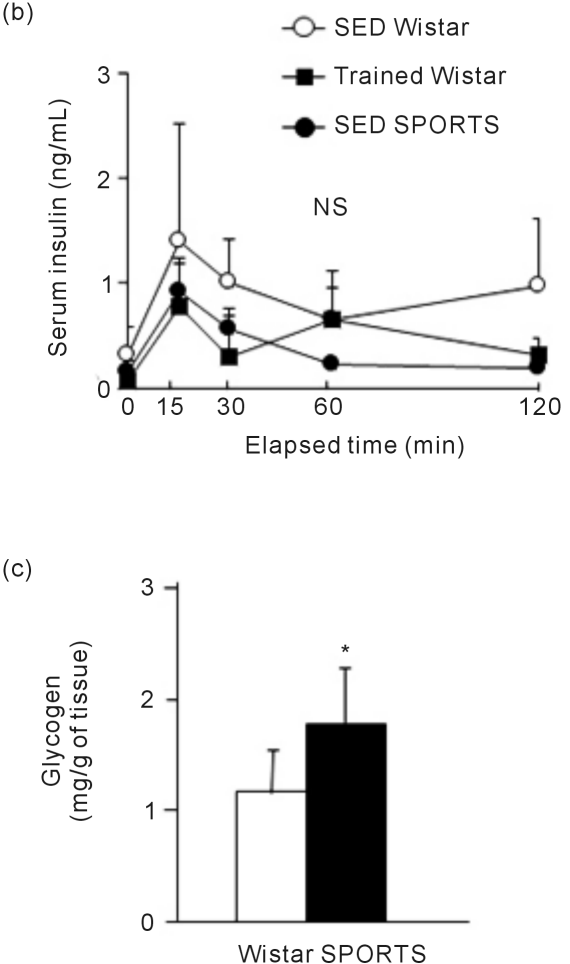

Figure 3. Glucose tolerance. (a) Blood glucose and (b) Serum insulin concentrations in SED Wistar (open squares), Trained Wistar (filled squares) and SED SPORTS (filled circles) rats determined at indicated times after glucose administration. Data are means \pm SD $(\mathrm{n}=4) .{ }^{*} P<0.05$ compared with SED Wistar rats. (c) Amounts of glycogen in gastrocnemius muscles of SED SPORTS and SED Wistar rats. Data are means \pm SD $(n=4)$. ${ }^{*} P<0.05$ compared with Wistar rats. EDL: extensor digitorum longus muscle; NS: not significant; SED: sedentary; SOL: soleus muscle.

SPORTS rats throughout the experimental period (Figure 3(a)). Serum insulin levels during glucose tolerance tests were slightly lower in SED SPORTS than in SED Wistar rats but did not significantly differ among groups (Figure 3(b)). We also measured amounts of glycogen in the gastrocnemius muscles of SED SPORTS and SED Wistar rats. The amount of muscle glycogen was significantly higher in SED SPORTS, than in SED Wistar rats (Figure 3(c)), suggesting that 
better insulin sensitivity was involved in the increased amount of glycogen in muscle of SPORTS rats. We used DNA microarray analysis to further identify genes contributing to glucose tolerance in SOL muscles from SED Wistar, Trained Wistar and SED SPORTS rats. We identified 140 exercise-sensitive genes with $>2$-fold increased expression in the muscles of Trained Wistar compared with SED Wistar rats. We further compared 116 genes with $>2$-fold increased expression between SED SPORTS and SED Wistar rats. Only 19 genes were upregulated in both Trained Wistar and SED SPORTS rats compared with SED Wistar rats (Figure 4(a)). We selected candidate genes involved in the better glucose tolerance in SPORTS rats among 97 genes with upregulated expression in SED SPORTS, but not Trained Wistar rats. We then focused on the erb-b2 receptor tyrosine kinase 3 (Erbb3) gene (Figure $4(\mathrm{~b})$ ). Erbb3 protein

(a) $\quad>2$-fold induction by training (trained Wistar vs. SED Wistar total 140 genes) $>2$-fold induction in SPORTS rats (SED SPORTS vs. SED Wistar, total 116 genes)

(b)

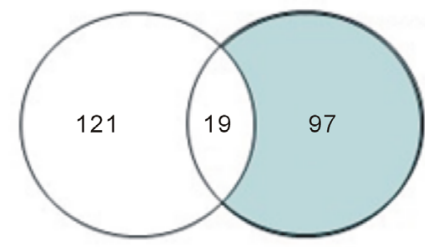

UniGene ID Fold Change Gene Description

\begin{tabular}{|c|c|c|}
\hline Rn.48677 & 6.199657 & TFN-induced protein \\
\hline Rn. 218880 & 4.652234 & Serine hydrolase-like 2 \\
\hline Rn.87496 & 4.611077 & Eosinophil-associated, ribonuclease A family, member 4 \\
\hline-- & 3.881638 & Myosin, heavy polypeptide $8, \mathrm{MyHC} 8$ \\
\hline Rn.9965 & 3.501402 & Troponin T2 \\
\hline Rn.24628 & 3.293894 & Leukocyte immunoglobulin-like receptor, subfamily $\mathrm{B}$, member 4 \\
\hline Rn. 13778 & 3.155575 & Osteoactivin \\
\hline Rn. 118271 & 3.109758 & Similar to immunoreceptor Ly $49 \mathrm{si} 3$ \\
\hline Rn.25754 & 3.034552 & TIMP 1 \\
\hline Rn.30516 & 2.9789 & Periostin \\
\hline Rn. 11333 & 2.828712 & Cellular retinoic acid binding g protein 2 \\
\hline Rn.764 & 2.746919 & Lectin \\
\hline Rn.3789 & 2.737558 & Ankyrin repeat domain 1 \\
\hline Rn. 90340 & 2.683725 & Resistin-like gamma \\
\hline $\mathrm{Rn} .137522$ & 2.683271 & C-type lectin domain family 4 , member a3 \\
\hline -- & 2.636819 & Similar to RIKEN cDNA $1110002 \mathrm{H} 13$ \\
\hline Rn.218406 & 2.396148 & Hypothetical protein LOC685699 \\
\hline Rn. 98430 & 2.389617 & Tubulin, b6 \\
\hline Rn.137139 & 2.32345 & Schlafen 8 \\
\hline Rn.142768 & 2.264991 & Olfactory receptor 756 \\
\hline Rn.86413 & 2.254877 & a-2u globulin PGCL4 \\
\hline -- & 2.2495184 & Similar to Epoxide hydrolase 1 \\
\hline Rn.167336 & 2.225651 & Proline rich Gla (G-carboxyglutamic acid) 4 (transmembrane) \\
\hline Rn.33323 & 2.2155 & Fc receptor, IgG, Iow affinity IIb \\
\hline Rn.9772 & 2.208626 & Complement component 3 a receptor 1 \\
\hline Rn.20787 & 2.195368 & Hypothetical protein LOC685203 \\
\hline Rn.141741 & 2.191617 & Olfactory receptor 295 \\
\hline -- & 2.188262 & Similar to lin-28 homolog b \\
\hline Rn.65764 & 2.181033 & GLI pathogenesis-related 1 (glioma) \\
\hline Rn.10228 & 2.172919 & Erbb 3 \\
\hline Rn.54397 & 2.155222 & Metallothionein $1 \mathrm{a}$ \\
\hline Rn.32351 & 2.145668 & Apolipoprotein E \\
\hline Rn.18560 & 2.145343 & Secretory leukocyte peptidase inhibitor \\
\hline Rn.3664 & 2.144139 & 4-Hydroxyphenylpyruvic acid dioxygenase \\
\hline Rn. 54397 & 2.117812 & Metallothionein $1 \mathrm{a}$ \\
\hline Rn.35322 & 2.101337 & Membrane bound O-acyltransderase domain containing 2 \\
\hline Rn.29233 & 2.069215 & S100 calcium binding protein $\mathrm{A} 3$ \\
\hline Rn.195462 & 2.059266 & Similar to $60 S$ ribosomal protein L29 (P23) \\
\hline Rn.10352 & 2.054423 & Cytochrome P 450, family 1 , subfamily a, polypeptide 1 \\
\hline Rn.3402 & 2.051285 & Sodium channel, voltage-gated, type III, b \\
\hline Rn.12746 & 2.048371 & LOC362793 \\
\hline Rn. 8273 & 2.043744 & Branched chain aminotransferase 1 , cytosolic \\
\hline
\end{tabular}

EST: 55 items 

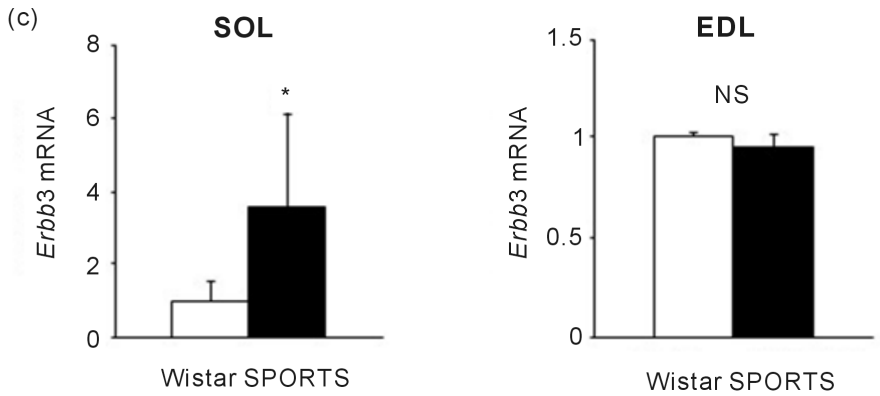

Figure 4. Candidate gene for better glucose tolerance in SPORTS rats. (a) Classification of exercise-sensitive and SPORTS rat-specific genes using DNA microarray analysis. Ratios of 140 genes were $>2$-fold higher in SOL muscle of Trained Wistar, compared with SED Wistar rats, indicating that these genes are exercise-sensitive. The ratios of 116 genes were $>2$-fold higher in SED SPORTS, than in SED Wistar rats, indicating that these were specific to SPORTS rats. Only 19 genes were common between exercise-sensitive and SPORTS rat-specific genes. (b) Annotation of SPORTS rat-specific genes. Gene ontology analyzed using Subio Platform Software. (c) Expression of Erbb3 mRNA in skeletal muscles of sedentary SPORTS and Wistar rats. Data are shows as means \pm SD $(n=7)$. Expression in sedentary Wistar rats is expressed as 1.0 . ${ }^{*} P<0.05$ compared with Wistar rats. EDL: extensor digitorum longus muscle; NS: not significant; SED: sedentary; SOL: soleus muscle.

binds neuregulin and stimulates glucose transport in skeletal muscle cells [14]. We confirmed that Erbb3 mRNA expression was upregulated in the SOL muscle of SED SPORTS rats compared with SED Wistar rats, but it was essentially identical in the EDL muscles of SED Wistar and SED SPORTS rats (Figure 4(c)).

\subsection{Lipid Oxidation in Skeletal Muscle of SPORTS Rats}

We evaluated lipid oxidation in the skeletal muscle of SED SPORTS rats, since they had less epididymal fat than SED Wistar rats despite similar body weight and food intake [2]. We found significantly more activity of $\beta$-HAD, a key lipid oxidation enzyme, in the SOL muscles in SED SPORTS, than in SED Wistar rats (Figure 5(a)). The $\beta$-HAD activity in EDL muscles was slightly higher in SED SPORTS, than in SED Wistar rats (Figure 5(a)). Comprehensive gene expression analyses revealed that the expression of genes associated with mitochondrial $\beta$-oxidation did not increase in the SOL muscle of SED SPORTS compared with SED Wistar rats (Figure 5(b)).

\section{Discussion}

Glucose tolerance was better in the SPORTS line of rats established by Morishima-Yamato et al. and they had less fat than Wistar rats [1]. The present study investigated the expression of genes contributing to glucose and fat metabolism in the skeletal muscle of SPORTS rats to account for their metabolic characteristics. The amount of expressed D-loop region was lower in the SOL muscles, but not the EDL muscles of SED SPORTS, compared with SED Wistar rats (Figure 1(a)), suggesting that the SOL muscles of SED SPORTS rats had impaired 
(a)

SOL

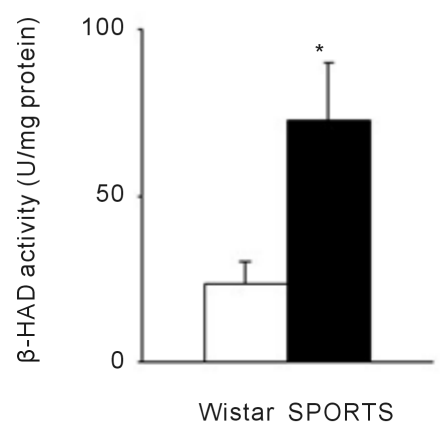

EDL

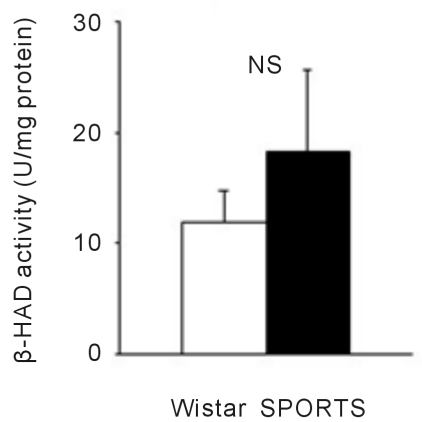

(b)

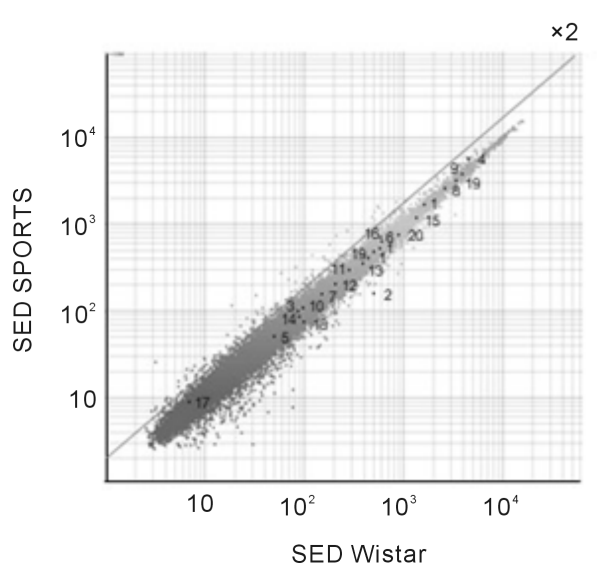

1 Acyl-Coenzyme Adehydrogenase, short chain/branched chain/very long chain 2 Adlponectin

3 Carnitne paimitoyltransferase $1 \alpha$

4 Fatty acid binding protein 3

5 3-Hydroxybutyrate

dehydrogenase. type 2

6 Hydroxyateroid $(17-\beta)$ dehydrogenase 4

7 Malonyl-CoA decarboxylase

8 Hydroxyacyl-Co A dehydrogenase, $\beta$-aubunit

9 Acyl-Coenzyme A dehydrogenase,

medium chain

10 Peroxisome biogenesis factor 7

11 PPAR $\delta$

12 Acyl-Coenzyme A oxidase 3, pristanoyl

$13 \mathrm{FK} 50 \delta$ binding protein 12-rapamycin

14 associate protein 1

Acyl-CoA oxidase 2, branched chain

15 Enoyl CoA hydratase, ahort chain, 1

16 Dodecenoyl-CoA סisomerase

17 Fatty acid binding protain 1

18 Enoyl-CoA hydratase/3-hydroxyacyl CoA

dehydrogenase

19 Hydroxyacyl-CoA dehydrogenase, $\alpha$-subunit

20 Carnitine paimitoyltransferase 2

Figure 5. Enzyme activity of mitochondrial fatty acid oxidation. (a) Enzymatic activity of $\beta$-hydroxyacyl-CoA dehydrogenase $\beta$-HAD in skeletal muscles of sedentary SPORTS and Wistar rats. Data are means $\pm \mathrm{SD}(\mathrm{n}=3) .{ }^{*} P<0.05$ compared with Wistar rats. (b) Expression of genes associated with lipid oxidation in SOL muscle of SED SPORTS and SED Wistar rats analyzed using DNA microarrays. Genes with similar expression lie on line from origin to top right corner, and amount of expression is indicated by distance from origin. Scale on each axis is log scale. Numbers indicate expression of respective lipid oxidation-associated genes listed in this panel. EDL: extensor digitorum longus muscle; NS: not significant; SED: sedentary; SOL: soleus muscle.

mitochondrial biogenesis. However, genes encoding mitochondrial respiratory chain enzymes ATPase 6 and $C O X 6 c$ were upregulated in the SOL, but not in the EDL muscles of SED SPORTS compared with SED Wistar rats. The respiratory activity per mitochondrion might be augmented via the transactivation of these genes in SED SPORTS rats. The amounts of Erbb3 mRNA expression and enzyme activity of $\beta$-HAD were also increased in the SOL, but not in the EDL muscles of SPORTS rats even when sedentary, suggesting that the skeletal muscle of SPORTS rats could utilize glucose and fat more rapidly than control Wistar rats. That is, the skeletal muscle, especially slow type SOL muscle, of SPORTS rats congenitally possesses higher metabolic activity, leading them to hyperactiv- 
ity when housed with running wheels.

Both PGC- $1 \alpha$ and PGC- $1 \beta$ regulate the expression of genes that encode proteins involved in the mitochondrial respiratory chains of skeletal muscle cells including Mt-Atp6 and COX6c [13] [15]. Significantly more Pgc-1a mRNA was expressed in the SOL muscles of SED SPORTS, than SED Wistar rats (Figure $1(\mathrm{~d})$ ). More $P g c-1 \beta$ transcripts were found in the EDL muscle of SED SPORTS, than SED Wistar rats, whereas expression levels were similar in the SOL of both groups (Figure 1(e)). Both PGC-1 $\alpha$ and PGC- $1 \beta$ might play distinct roles in regulating the expression of these genes between slow- and fast-twitch skeletal muscles.

The metabolic properties of skeletal muscles are reflected in the composition of the myofibers, and PGC-1 $1 \alpha$ and PGC- $1 \beta$ drive the formation of slow-oxidative myofibers via the upregulation of type I and IIa, and highly oxidative type IIx myofibers, respectively [16] [17]. However, gene expression profiles of $P g c-$ $1 \alpha$ and $P g c-1 \beta$ could not explain the mechanism of the increased ratio type IIa myofibers in the EDL muscles of SED SPORTS rats. Signaling pathways that function to transactivate type IIa myosin independently of PGCs might be essential for the shift of fiber type in the EDL muscles of SED SPORTS rats.

The amounts of blood glucose and muscle glycogen were lower and higher, respectively, in SED SPORTS, than SED Wistar rats (Figure 3(a) and Figure $3(c)$ ). We analyzed gene expression to identify candidate genes contributing to the better glucose tolerance in the SPORTS rats. Our comprehensive DNA microarray analysis revealed that the upregulated genes in the SOL muscles of SED SPORTS rats were very different from the training-sensitive genes which were upregulated in Wistar rats. The mechanisms of glucose tolerance also seemed to differ between SPORTS and trained Wistar, even though exercise improved glucose tolerance [18]. We also found upregulated Erbb3 mRNA expression in the SOL muscle of SPORTS, but not in Trained Wistar rats (Figure 4(b)). Erbb3 is a potent receptor for neuregulin, which enhances the translocation of glucose transporter 4 to the surface of muscle cells independently of insulin signaling [14]. The upregulation of Erbb3 could be one mechanism of the better glucose tolerance in SED SPORTS rats.

The lower fat mass in SED SPORTS compared with SED Wistar rats implies impaired triglyceride synthesis or enhanced lipid oxidation in the skeletal muscle of SED SPORTS rats. The elevated activity of $\beta$-HAD in SED SPORTS than SED Wistar rats indicated that mitochondrial fatty acid oxidation is increased in the SOL muscles of SED SPORTS rats (Figure 5(a)). However, the expression of genes associated with mitochondrial $\beta$-oxidation did not increase in the SOL muscle of SED SPORTS rats compared with SED Wistar rats (Figure 5(b)), indicating that the amounts of transcripts did not contribute to the increase in the $\beta$-HAD activity of SED SPORTS rats. The enzymes associated with $\beta$-oxidation might undergo posttranscriptional modification in the SOL muscles of SED SPORTS rats. 
The present study showed that unique gene expression in the skeletal muscle (especially SOL) of SPORTS rats even when sedentary allows more rapid glucose and fatty acid utilization, leading to hyperactive running. We identified only 19 genes that were commonly upregulated in Trained Wistar and SED SPORTS rats, suggesting that the accelerated metabolic properties of SPORTS rats differ from those that are upregulated by exercise. However, we could not identify an absolute requirement of $E r b b 3$ for better glucose tolerance and the mechanism to increase $\beta$-HAD activity in the skeletal muscles of SED SPORTS rats. Further investigation is required to elucidate these mechanisms and uncover novel approaches to improving metabolic disorders without the need for extreme exercise.

\section{Acknowledgements}

This study was implemented as part of JSPS KAKENHI to T. Nikawa (JP15H04960, 16H01645) from the Ministry of Education, Culture, Sports, Science, and Technology (MEXT) of Japan.

\section{References}

[1] Morishima-Yamato, M., Hisaoka, F., Shinomiya, S., Harada, N., Matoba, H., Takahashi, A. and Nakaya, Y. (2005) Cloning and Establishment of a Line of Rats for High Levels of Voluntary Wheel Running. Life Sciences, 77, 551-561. https://doi.org/10.1016/j.lfs.2004.10.074

[2] Hattori, A., Mawatari, K., Tsuzuki, S., Yoshioka, E., Toda, S., Yoshida, M. and Nakaya, Y. (2010) $\beta$-Adrenergic-AMPK Pathway Phosphorylates Acetyl-CoA Carboxylase in a High-Epinephrine Rat Model, SPORTS. Obesity, 18, 48-54. https://doi.org/10.1038/oby.2009.145

[3] Pieter, L., Moreno, M., Silvestri, E., Lombardi, A., Goglia, F. and Lanni, A. (2007) Fuel Economy in Food-Deprived Skeletal Muscle: Signaling Pathways and Regulatory Mechanisms. The FASEB Journal, 21, 3431-3441. https://doi.org/10.1096/fj.07-8527rev

[4] Schiaffino, S. and Reggiani, C. (2011) Fiber Types in Mammalian Skeletal Muscles. Physiological Reviews, 91, 1447-1531. https://doi.org/10.1152/physrev.00031.2010

[5] Mizunoya, W., Wakamatsu, J.I., Tatsumi, R. and Ikeuchi, Y. (2008) Protocol for High-Resolution Separation of Rodent Myosin Heavy Chain Isoforms in a Mini-Gel Electrophoresis System. Analytical Biochemistry, 377, 111-113. https://doi.org/10.1016/j.ab.2008.02.021

[6] Nikawa, T., Ishidoh, K., Hirasaka, K., Ishihara, I., Ikemoto, M., Kano, M., Kominami, E., Nonaka, I., Ogawa, T., Adams, G.R., Baldwin, K.M., Yasui, N., Kishi, K. and Takeda, S. (2004) Skeletal Muscle Gene Expression in Space-Flown Rats. The FAS EB Journal, 18, 522-524. https://doi.org/10.1096/fj.03-0419fje

[7] http://www.subio.jp/

[8] Gao, X., Zhao, X.L., Zhu, Y.H., Li, X.M., Xu, Q., Lin, H.D. and Wang, M.W. (2011) Tetramethylpyrazine Protects Palmitate-Induced Oxidative Damage and Mitochondrial Dysfunction in C2C12 Myotubes. Life Sciences, 88, 803-809. https://doi.org/10.1016/j.lfs.2011.02.025

[9] Coderre, L., Vallega, G.A., Pilch, P.F. and Chipkin, S.R. (2007) Regulation of Gly- 
cogen Concentration and Glycogen Synthase Activity in Skeletal Muscle of InsulinResistant Rats. Archives of Biochemistry and Biophysics, 464, 144-150. https://doi.org/10.1016/j.abb.2007.04.012

[10] Holloway, G.P., Lally, J., Nickerson, J.G., Alkhateeb, H., Snook, L.A., Heigenhauser, G.J., Calles-Escandon, J., Glatz, J.F., Luiken, J.J., Spriet, L.L. and Bonen, A. (2007) Fatty Acid Binding Protein Facilitates Sarcolemmal Fatty Acid Transport But Not Mitochondrial Oxidation in Rat and Human Skeletal Muscle. Journal of Physiology, 582, 393-405. https://doi.org/10.1113/jphysiol.2007.135301

[11] Hood, D.A. (2009) Mechanisms of Exercise-Induced Mitochondrial Biogenesis in Skeletal Muscle. Applied Physiology, Nutrition, and Metabolism, 34, 465-472. https://doi.org/10.1139/H09-045

[12] Dimauro, S. and Schon, E.A. (2003) Mitochondrial Respiratory-Chain Diseases. New England Journal of Medicine, 348, 2656-2668. https://doi.org/10.1056/NEJMra022567

[13] Wu, Z., Puigserver, P., Andersson, U., Zhang, C., Adelmant, G., Mootha, V., Troy, A., Cinti, S., Lowell, B., Scarpulla, R.C. and Spiegelman, B.M. (1999) Mechanisms Controlling Mitochondrial Biogenesis and Respiration through the Thermogenic Coactivator PGC-1. Cell, 98, 115-124.

[14] Cantó, C., Suárez, E., Lizcano, J.M., Griñó, E., Shepherd, P.R., Fryer, L.G., Carling, D., Bertran, J., Palacín, M., Zorzano, A. and Gumà, A. (2004) Neuregulin Signaling on Glucose Transport in Muscle Cells. Journal of Biological Chemistry, 279, 12260 12268. https://doi.org/10.1074/jbc.M308554200

[15] Lin, J., Puigserver, P., Donovan, J., Tarr, P. and Spiegelman, B.M. (2002) Peroxisome Proliferator-Activated Receptor $\gamma$ Coactivator $1 \beta(P G C-1 \beta)$, a Novel $P G C-1$ Related Transcription Coactivator Associated with Host Cell Factor. Journal of Biological Chemistry, 277, 1645-1648. https://doi.org/10.1074/jbc.C100631200

[16] Spangenburg, E.E. and Booth, F.W. (2003) Molecular Regulation of Individual Skeletal Muscle Fibre Types. Acta Physiologica Scandinavica, 178, 413-424. https://doi.org/10.1046/j.1365-201X.2003.01158.x

[17] Arany, Z., Lebrasseur, N., Morris, C., Smith, E., Yang, W., Ma, Y., Chin, S. and Spiegelman, B.M. (2007) The Transcriptional Coactivator $P G C-1 \beta$ Drives the Formation of Oxidative Type IIX Fibers in Skeletal Muscle. Cell Metabolism, 5, 35-46. https://doi.org/10.1016/j.cmet.2006.12.003

[18] Perseghin, G., Price, T.B., Petersen, K.F., Roden, M., Cline, G.W., Gerow, K., Rothman, D.L. and Shulman, G.I. (1996) Increased Glucose Transport-Phosphorylation and Muscle Glycogen Synthesis after Exercise Training in Insulin-Resistant Subjects. New England Journal of Medicine, 335, 1357-1362. https://doi.org/10.1056/NEJM199610313351804 